\title{
Design of Wireless Anti-spoofing Device for Measuring Blood Oxygen Concentration and Heart Rate
}

\author{
Vipin Gupta \\ PEC University of Technology \\ Chandigarh, India
}

\author{
Mandeep Singh \\ Engineer, DEC Division \\ C-DAC, Mohali, Punjab India
}

\author{
Rita Mahajan \\ PEC University of Technology \\ Chandigarh, India
}

\begin{abstract}
This project describes to design anti-spoofing device which measures continuously heart rate, blood oxygen concentration and record the data of the patient on PC screen serially. When the data of the patient goes below the critical value, the monitoring system automatically transmits the information to the doctor's hand phone on the mobile network as a SMS via a GSM device. Thus it's alert to doctor to quickly attend the patient and give diagnosis to the patient's. This project made the device economical and also creates the easy pathway between the patient and doctor which provide better health care facility to patient.
\end{abstract}

\section{Keywords}

Pulse Rate, $\mathrm{SpO}_{2}$, Microcontroller, PC, LM358, ADC, GSM modem

\section{INTRODUCTION}

Pulse-oximeter is a non-invasive device uses two frequencies of light (red and infrared) to determine the percentage $(\%)$ of hemoglobin in the blood that is saturated with oxygen. The percentage is called blood oxygen saturation or $\mathrm{SpO}_{2}$. It also measures pulse rate at the same time. Pulse-Oximeter is based on measuring the absorption of red and infrared light that passes a patient finger by using light sensors .Absorption on each wavelength differs significantly for the oxygenated hemoglobin and deoxygenated hemoglobin. The oxygenated hemoglobin allows red right to transmit through and absorbs more infra red light while the deoxygenated hemoglobin allows infrared to transmit through and absorbs more red light as shown in Fig.1

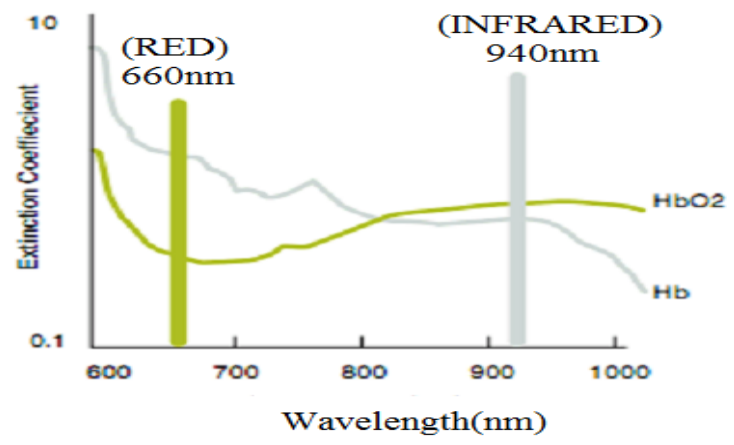

Figure 1: The absorption levels of oxygenated and deoxygenated blood at different wavelength [13]

The absorption of light by $\mathrm{HbO}_{2}$ or $\mathrm{Hb}$ varies with the pulsatile flow of blood into the tissues, so the absorption varies with the same frequency as the heart beat. With each heart beat, the volume of arterial blood in the tissue increases, while the remaining tissue volume remains constant. This change makes it possible for the oximetry system to differentiate the arterial blood from all other absorbing substance has both DC and AC components in it; this is as shown in Fig 2.

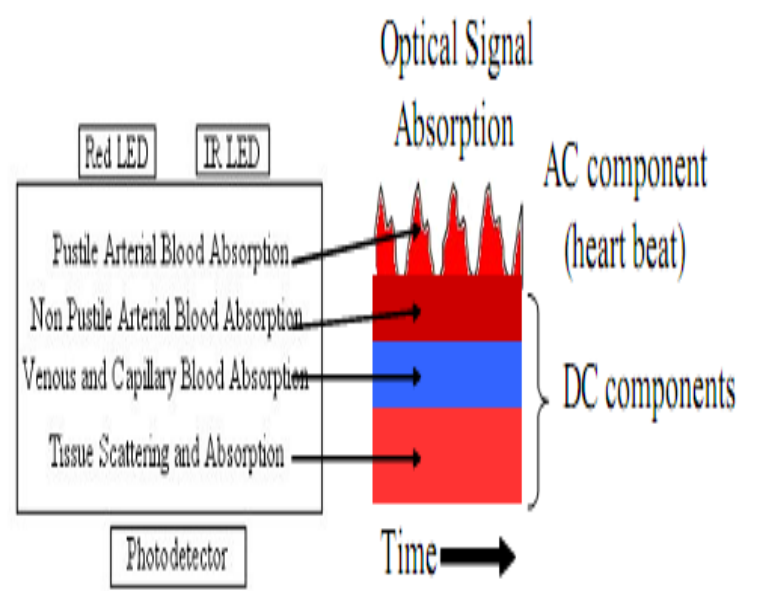

Figure 2: AC and DC components of oximetry [14].

The time varying component or the $\mathrm{AC}$ part in the absorbance spectrum is due to the arterial injection or fresh oxygenated blood. The steady DC part in the spectrum is caused by the absorbance of light by the tissue, skin and bone. The DC part has no information in determining the oxygen saturation. Only the $\mathrm{AC}$ component is the pulsatile waveform which is of our interest. On the basis of the values of $\mathrm{AC}$ and $\mathrm{DC}$ values of the two frequencies gives the Oxygen saturation. LED's are used as a light source and sequentially pulsed at a fast rate. To find Oxygen saturation, first calculate $\mathrm{R}$ which is absorbance ratio of the arterial blood at the two wavelength is then determined as the ratio of $\mathrm{AC}$ variation, each normalize to their DC values.:

$\mathrm{R}=\left(\mathrm{AC} \_\right.$red $\div \mathrm{DC} \_$red $) \div\left(\mathrm{AC} \_\mathrm{IR} \div \mathrm{DC} \_\mathrm{IR}\right)$

AC_red and AC_IR are the AC components of red and infrared light source .DC_red and DC_IR are the DC component of red and infrared light sources respectively.

The formula to find oxygen saturation:

$\mathrm{SaO}_{2}=\mathrm{A}-\mathrm{B} * \mathrm{R}$

While A and B component are two constants, as soon as we get the values of AC and DC component of red and infrared LED. The blood oxygen saturation can be getting very easily by the formula. 


\section{GSM Module}

The modem used for this work is SIM 900. This modem is a highly flexible plug and play GSM 900 / 1800 / 1900 Tri Band usage with RS232 interfaces. It's designed for GPRS, Fax, SMS, Data and voice applications. It has a built in TCI / IP and RTC feature. It is standard AT Commands operated. This modem has been used and tested in VTS, vehicle and home security Products, M2M applications, PLCs, Pump controllers, Display boards etc. Now this modem is used in medical Industry for providing a better health care facility to patient.. In market $5 \mathrm{~V}$ and $12 \mathrm{~V}$ input power supply models available. RF antenna is connected via SMA connector. It is a Low cost, compact and very easy to use modem [15]

\section{HARDWARE DESIGN}

Pulse Oximeter block diagram as shown in Fig 3 is.

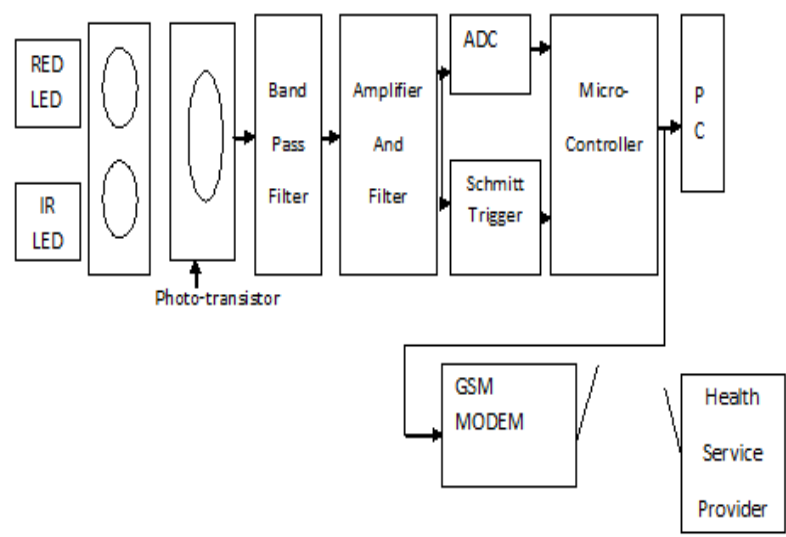

Figure 3: Block Diagram of Pulse oximeter

Two methods exist to design the sensor that can detect the blood absorption:

1. Transmission/forward scattered model: In the forward scattered model, the LED's and the photo-transistor are situated in the opposite side of the body

2. Reflection/ backscattered model: In the backscattered model, the LED's and the photo-transistor exist on the same side of the body.

The hardware system for pulse-oximeter measurement device consists of figure clip, Red LED, Infrared LED, PhotoTransistor, LM358, ADC and the main control system based on 89C51 like Fig 4.

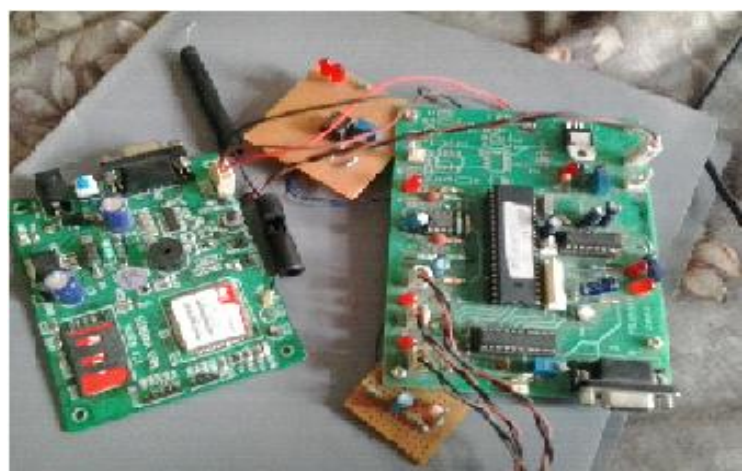

Figure 4: Hardware system For Pulse Oximeter
The sensor arrangement used in the transmittance method in which sensor is placed on the finger consists of two LED and a photo-detector as shown in Fig 5.

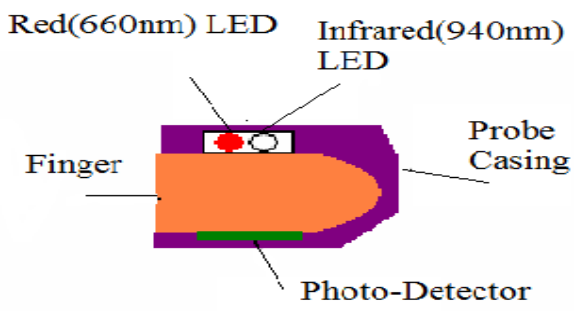

Fig 5: Sensor orientation for light transmittance [2]

The two LED used in the sensor part operating at different wavelengths; one red LED with a wavelength of $660 \mathrm{~nm}$, the other, an infrared LED with a wavelength of $940 \mathrm{~nm}$. The LEDs are designed to be placed opposite of photo-detector that detects the light from the LED. The two wavelengths are chosen because de-oxygenated hemoglobin has a higher absorption at around $660 \mathrm{~nm}$ and at $940 \mathrm{~nm}$ oxygenated hemoglobin has higher absorption.

The LEDs switches on and off alternatively. The two LEDs never shine simultaneously. The receiver was used to sense the light attenuated by body tissue and covert the optical intensity into voltage. The signal collected from the photodetector (photo-transistor) was supplied to the band pass filter with pass band of 0 to $2.5 \mathrm{~Hz}$. These frequencies with in this range are required since the lowest human rate can be approximately 30 beats per minute and the highest around 200 beats per minute. The pulsatile changes in transmitted light were very weak, and so the amplification stage was necessary. The signal was amplified two times having each stage gain of 100 was provided using LM358 after passing through filter circuitry. The circuit consists of two stage high gain; active low pass filter with a overall gain of the two stages was 10000 having a cut off frequency of $0-2.5 \mathrm{~Hz}$. A capacitor was used at the input to remove DC component between each stage of amplification. The output obtained after amplification does not contain DC component. The amplified waveform was feed to ADC which convert analog signal to digital and the same output obtained after amplification was given to the Schmitt trigger which convert pulses into square wave.

\section{MICROCONTROLLER}

Microcontroller controls all operation to calculate oxygen saturation and heart rate and transmit the patient information on PC serially and also transmit the patient information to doctor by SMS when the set condition of the patient goes false. The sensor output was in analog form so the signal is fed to analog to digital converter where the analog signal is digitized. The ADC is controlled by microcontroller. The digitized output is used to calculate oxygen saturation. For calculating oxygen saturation and pulse rate and transmit the patient data on PC and transmit the patient information to the doctor by the SMS, a software program was developed. The program executing on the microcontroller does the following actions:

1. Initialize the serial communication parameters and the GSM modem by send the command. 
2. Turn on and off Both the Led alternatively.

3. The corresponding output from LEDs is digitize by ADC and is stored in RAM.

4. Find the maximum value of the corresponding LED and is stored in RAM

5. Microcontroller mathematically divides the data from both sensors i.e.

$\mathrm{R}=\mathrm{V}_{\text {RED_MAX }} \div \mathrm{V}_{\text {IR_MAX }}$.

6. The program can calculate oxygen saturation according equation (2).To get the value of $\mathrm{A}$ and $\mathrm{B}$ we need a tool called oxygen saturation simulator. A and B coefficient that are determined when the Pulse-Oximeter is being calibrated. After calibration the value obtained of A and $\mathrm{B}$ and $\mathrm{R}$ put in the formula so the oxygen saturation can be determined. The results obtained are stored in RAM and also displayed on PC.

7. For calculation Pulse Rate, only IR-LED was used. Where, the number of pulses is counted for 10 second and multiply by 6 gives pulse rate.

\section{Pulse rate $=$ Count $* 6$}

The results obtained are stored in RAM and displayed on PC.

8. The results obtained after the microcontroller had done computational work to find oxygen saturation and pulse rate. Microcontroller checks the results obtained with the set condition. If the anyone of the set condition is false then it activate the GSM Modem and send the information of patient to doctor wirelessly Via SMS.

9. Microcontroller continuously monitors blood oxygen concentration and Pulse rate and results are displayed on PC continuously.

\section{TESTING}

Initially the sensor was designed with the reflectance method. This method causes a large noise and the output contained spikes and missing data due to the movement of the finger. Moreover, the design was uncomfortable, hampered practicality to a large extent and did not provide stability for the development process. Therefore, the design was changed to a finger clip that will hold the photo-detector and the LED's tightly against the skin.

\section{RESULTS}

Results obtained showed a convincing conclusion that the system built can detect the heart beat and $\mathrm{SpO}_{2}$. The results can be obtained on the PC serially. The results of the patient are sent by SMS to doctor when the patient condition set goes False. The system uses the $89 \mathrm{c} 51$ as Microcontroller.

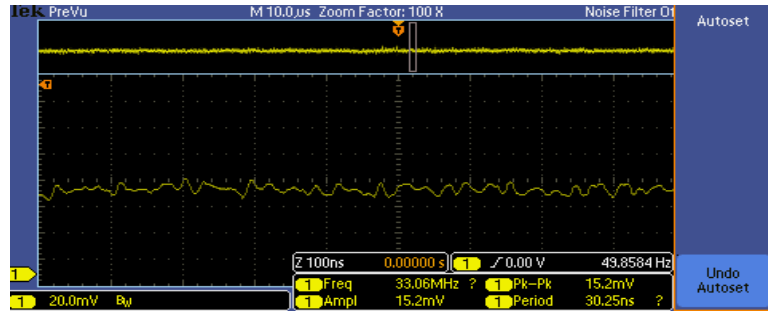

Figure 6: PPG waveform of IR-LED
The PPG output waveform as shown in Fig 6 is the signal output of the photo-detector when Infrared LED was ON for some time. The signal was very noisy and hence applied to a band pass filter. After attenuating noise present in the signal, the output obtained after removing noise is shown in Fig 7.

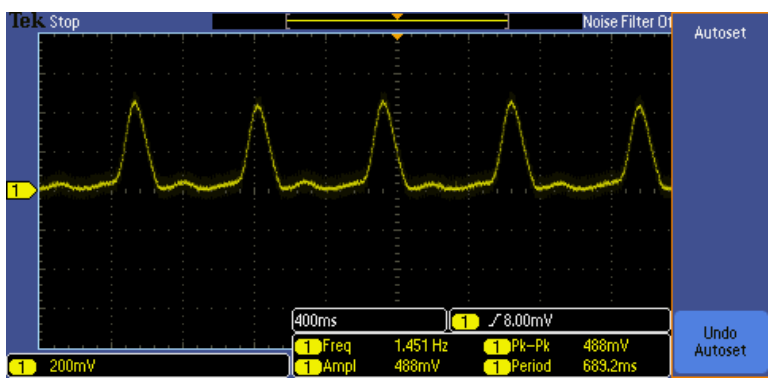

Figure 7. Filtered waveform of IR-LED

The amplitude of the signal is less than required so it was amplified. The waveform after amplification of the previous stage output is shown in Fig 8.

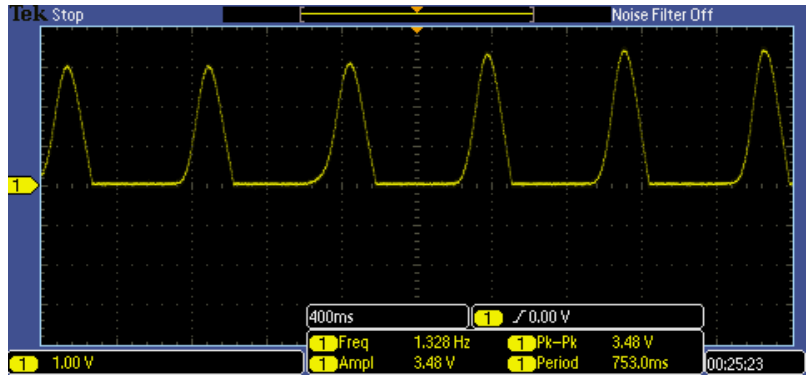

Figure 8: Amplified waveform of IR-LED

To calculate Pulse Rate, the signal was Fed to Schmitt Trigger to convert the waveform into a Square Wave. The waveform output of the Schmitt trigger is shown in Fig 9.

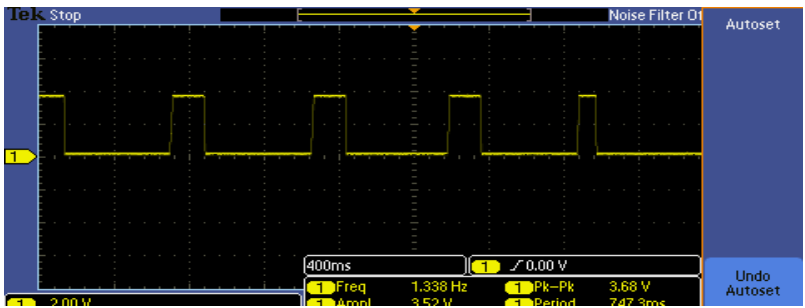

Figure 9: Digital waveform of IR-LED

The waveform obtained after amplification when Red LED is $\mathrm{ON}$ as shown in Fig10.

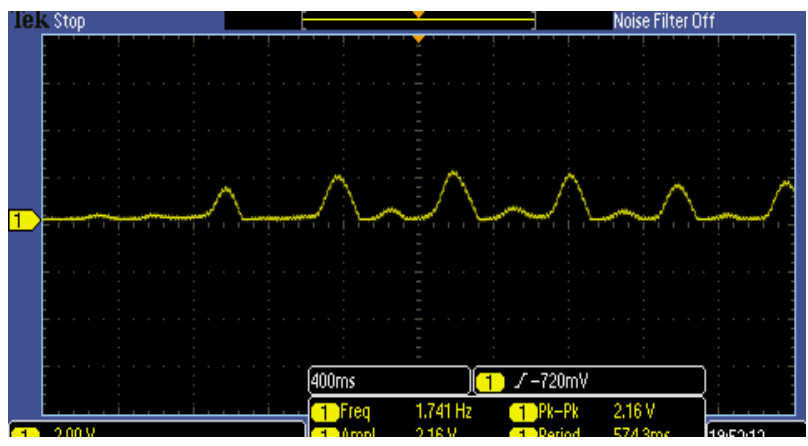

Figure 10: Amplified waveform of Red LED 
We compared the results of a clinically approved PulseOximeter to that of the one designed. We found that $\mathrm{SpO}_{2}$ readings are similar with a confidence interval of $98 \%$ where as in pulse rate error of around 2 beats per minute. Samples were taken from age group of around 20 to 60 . The results of this experiment are shown in Table 1 on various subjects. These subjects are healthy people. The results on PC are shown in figure 11, where reading between $\mathrm{S}$ and $\mathrm{O}$ was $\mathrm{SpO}_{2}$ reading and the reading between $\mathrm{P}$ and $\mathrm{R}$ was Pulse Rate. The critical value was set for showing in a demo for Blood Oxygen Concentration or $\mathrm{SpO}_{2}$ is 95 and for Pulse Rate is 100 , Whenever the blood oxygen concentration goes below the 95 or the heart rate goes above the 100 , the SMS is send to doctor Wirelessly via GSM Modem. The results on mobile phone as a SMS are shown in figure 12.

Table1 Measured $\mathrm{SpO}_{2}$ and pulse rate values

\begin{tabular}{|c|l|l|c|}
\hline $\begin{array}{l}\text { Measure } \\
\mathrm{SpO}_{2} \text { by } \\
\text { Pulse- } \\
\text { Oximeter } \\
(\%)\end{array}$ & $\begin{array}{l}\text { Measur } \\
\text { ed } \\
\mathrm{SpO}_{2} \\
(\%)\end{array}$ & $\begin{array}{l}\text { Measure } \\
\text { Pulse rate } \\
\text { By Pulse } \\
\text { Oximeter }\end{array}$ & Measured \\
\hline 98 & 99 & 78 & 80 \\
\hline 98 & 98 & 71 & 72 \\
\hline 98 & 97 & 106 & 105 \\
\hline 97 & 98 & 92 & 90 \\
\hline 98 & 98 & 56 & 58 \\
\hline 97 & 97 & 84 & 84 \\
\hline 94 & 93 & 98 & 96 \\
\hline
\end{tabular}

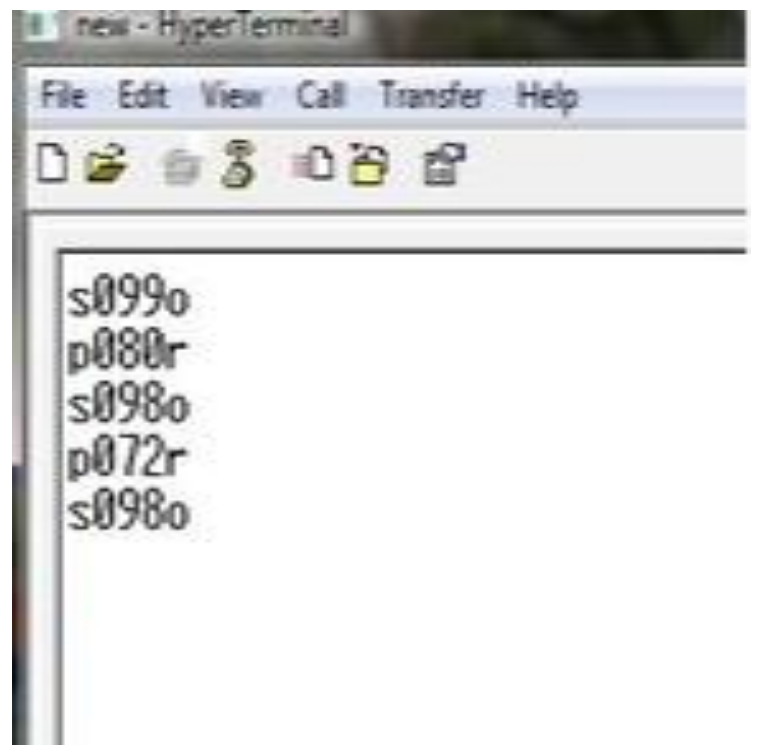

Figure 11: Results of Blood oxygen Concentration and Pulse Rate on PC.

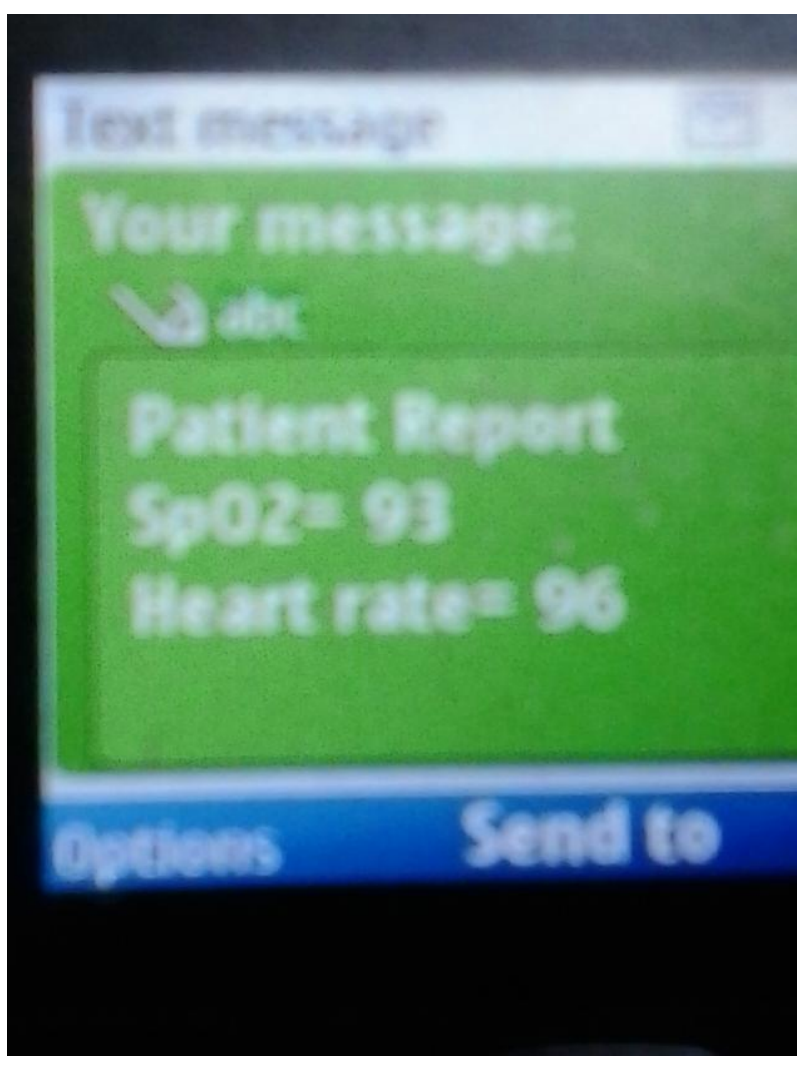

Figure 12: Snapshot of the send SMS.

.The results obtained of a single healthy individual from two systems are shown graphically as shown in figure 13 and 14 and also shown in table 2 . The comparison shows that in heart rate measurement has accuracy with a mean of $2.63 \%$ and $\mathrm{SpO}_{2}$ has a mean accuracy of $1.02 \%$.

Table 2: Measured $\mathrm{SpO}_{2}$ and Pulse Rate Values of a healthy Individual

\begin{tabular}{|c|c|c|c|}
\hline $\begin{array}{c}\text { Measured } \\
\mathrm{SpO}_{2} \text { by } \\
\text { Pulse-oximeter }\end{array}$ & $\begin{array}{c}\text { Measured } \\
\mathrm{SpO}_{2} \quad(\%)\end{array}$ & $\begin{array}{c}\text { Measured } \\
\text { Pulse rate by } \\
\text { PO }\end{array}$ & $\begin{array}{c}\text { Measured } \\
\text { Pulse Rate }\end{array}$ \\
\hline 98 & 99 & 70 & 68 \\
\hline 98 & 98 & 84 & 80 \\
\hline 97 & 97 & 74 & 76 \\
\hline 97 & 96 & 77 & 76 \\
\hline 98 & 98 & 71 & 78 \\
\hline 97 & 97 & 76 & \\
\hline
\end{tabular}




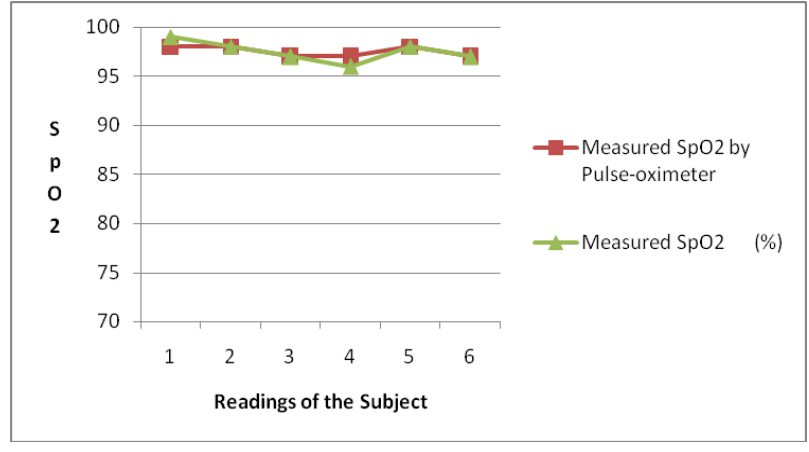

Figure 13 : Graphical representations of $\mathrm{SpO}_{2}$ readings of healthy Individual

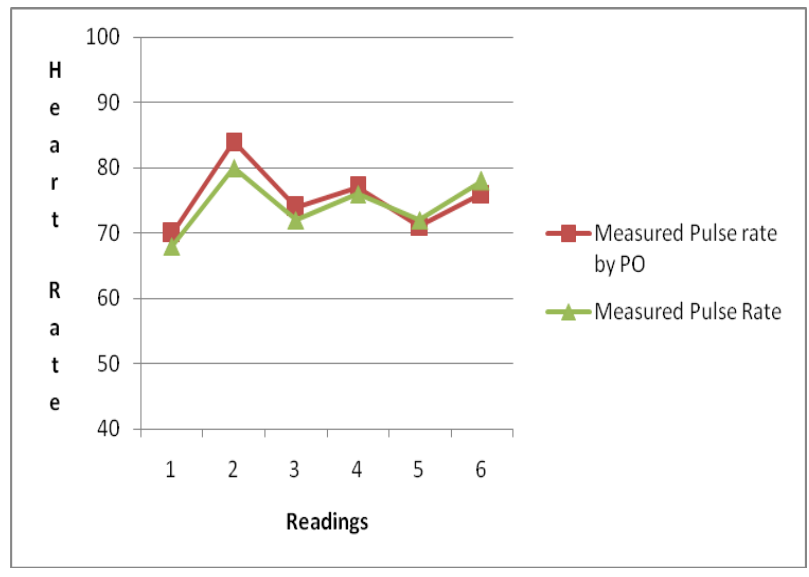

Figure 14 : Graphical representations of Pulse rate readings of healthy Individual

\section{FUTURE SCOPE}

The scope of development is endless but the authors would like to suggest the following areas where work can be done:

1. The Output can be feed to a local LCD.

2. The alarm can be added when heart beat measurement is done.

3. Can be added to bigger modules like Patient monitors.

4. It can be possible to add the feature to do the call to doctor when the patient condition is critical.

5. The patient information can be send via Email to the hospital server or the doctor Emai-id.

\section{CONCLUSION}

A system for continuous monitoring of patient using sensors which transmit data wirelessly through GSM modem has been designed, fabricated and tested. As the switch is ON first it measure blood oxygen level then pulse rate of a human body and send this data to PC serially via DB9. When the results obtained of the patient goes below the critical value, the information was sent successfully to the doctor. Assembly Language program is used in whole project. The developed system is user friendly for both patient and doctor and involves no cumbersome processes to operate. Overall the hardware and software implemented for this system successfully fulfils required goals.

\section{REFERENCES}

[1] Takahiro Asaoka and Kazushige Magatani, "Development of the device to detect human's biosignals by easy sensing", IEEE EMBS 2008W.-K. Chen, Linear Networks and Systems (Book style). Belmont, CA: Wadsworth, 1993, pp. 123-135

[2] Moyle, J. (2003). Pulse oximetry. Second edition, BMJ Publishing Group. London, United Kingdom.Journal of Anesthesia (ISBN 0-7279-1740-4) pp 174.

[3] P.C. Branche, W. S. Johnston, C. J. Pujary, and, Y. Mendelson,"Measurement Reproducibility and Sensor Placement Considerations in Designing a Wearable Pulse Oximeter for Military Applications," 30th Annual Northeast Bioengineering Conference, 2004.

[4] Deni, H. Muatore, D. M. Malkin, "Development of a Pulse Oximeter Analyzer for the Developing World" 31st Annual conferenceNortheast Bio-engineering Confe rence 2005

[5] F. Ferrero, F., Blanco, M., Blanco, ," Design of a lowcost instrument for pulse oximetry" Proceedings of the IEEE Instrumentation and Measurement Technology Conference, . 2006

[6] Yasuhiro Saeki, Komin Takamuraand Kazushige Magatani,"The measurement technique of human's bio-s signals", IEEE EMBS 2006B. Smith, "An approach to 415 graphs of linear forms (Unpublished work style)," Unpublished

[7] Guowei Di, Xiaoying Tang, Weifeng Liu, "A Reflectance Pulse Oximeter Design Using the MSP430OF149” IEEE International Conference on complex Medical Engineering, 2007

[8] Yoshiaki kanadea, Kazushige Magatani,'Development of device to detect $\mathrm{SpO} 2$ in the Field" 31st Annual International Conference of the IEEE EMBS Minneapolis, Minnesota, USA, September 2-6, 2009

[9] Chan-kyu Park, Jo-Chan Sohn,Jae-Hong Kim,Ho-Jin Choi,"Artifact-resistant design of a wrist-type heart rate monitoring device" 11th International Conference on Advanced Communication Technology, 2009.

[10] Cho Zin Myint, Nader Barsoum, Wong Kiing Ing, "Design a medicine device for blood oxygen concentration and heart rate", published in Global Journal of Technology and Optimization, Vol1, 2010

[11] Aziz, N.H.A. , Muhamad, W.N.W. , Wahab, N.A., "Real Time Monitoring Critical Parameters in Tissue Culture Growth Room with SMS Alert System", International Conference on Intelligent Systems, Modelling and Simulation(ISMS),2010 . .

[12] Zarka,N., Al-Houshi, Akhkobek, "Temperature Control Via SMS", International conference on Information and Communication Technologies, (ICICT ), 2010

[13] Santiago Lopez, "Pulse Oximeter Fundamental and Design”,Freescale Semiconductor Application Note with Document Number:AN4327,Rev.1,09/2011

[14] Dave Hoff,Roy Zhang, Tad Statter,Mike Carlson,'Pulse Oximetry.pdf',App_525_pro2

[15] http://www.gsmworld.com

[16] http://forum.gsmhosting.com

[17] http://surrey.ac.uk/pulseOximetry.pdf 